\title{
Effect of Ion Irradiation on the Mechanical Properties of High and Low Copper
}

\author{
H.M. Qadr \\ Department of Physics, College of Science, University of Raparin, Sulaimanyah, Iraqi Kurdistan, Iraq
}

\section{ARTICLE INFO}

Article history:

Received 29 December 2018

Received in revised form 14 February 2020

Accepted 18 February 2020

\section{Keywords:}

Irradiation

Hardness

dpa

Copper

\begin{abstract}
A B S T R A C T
An investigation into the effects of proton beam exposure on high- and low-copper structural materials for nuclear reactors has been carried out. The aim of this work was to investigate the impact of proton energy irradiation on the damage of the materials. The damage parameter used in the evaluation was displacement per atom (dpa) in material as a function of proton energy. In addition, a TRIM code was used to identify the penetration depth in response to changes in proton energy. The effect of proton beam exposure on the irradiation induced hardening of the different copper levels was investigated by Vickers Hardness tests for microstructural changes examination. The proton beam incident energy was $3 \mathrm{MeV}$ and the temperature was kept at approximately $30{ }^{\circ} \mathrm{C}$. A $25 \mu \mathrm{m}$ flat damage profile was achieved at 0.367 and $0.373 \mathrm{dpa}$ for low and high copper samples, respectively. The hardness variation with depth and yield strength variation with dose (dpa) were also investigated. Based on the results, the study found that the hardness test for the high copper was higher than the low copper.
\end{abstract}

\section{INTRODUCTION}

Ion irradiation experiments allow control of ion energy, dose, dose rate and temperature to obtain reproducible and specific results desirable for studying changes in irradiated micro-structure and property of a material in a shorter time [1-3]. However, the evolution of irradiated microstructure is dependent upon a combination of damage rate and irradiation temperature. Damage cannot always be reproduced in a relatively shorter time through increased displacement rate (dpa/s) alone, so temperature shift relations have to be created to allow for more correlation of one type of damage evolution from one irradiation environment to another using dose, dose rate and temperature [4]. One of the advantages of using proton beam is that parameters can be easily varied. Ion beams are also generally cheaper to produce a given dose compared to neutron sources.

Copper impurity has been found in older generation reactor pressure vessel (RPV) steels due

\footnotetext{
*Corresponding author.

E-mail address: hiwa.physics@uor.edu.krd DOI: https://doi.org/10.17146/aij.2020.923
}

to the use of copper coated welding rods [5]. It was found that 0.3 and $0.05 \mathrm{wt} \%$ were used for high and low impurity levels of copper, respectively. A study by Zhang et al. [6] suggests that copper precipitates that are rich in other impurities $(\mathrm{Ni}, \mathrm{Al}$ and $\mathrm{Mn}$ ) formed under proton irradiation remain stable with further irradiation. Clusters of these precipitates inhibit the movement of dislocations under stress, which causes a net increase in hardness after irradiation. The mechanism of irradiation hardening is similar to that of aging except that proton radiation increases the free movement of vacancies, which encourages the diffusion of solutes. Another similarity to thermal aging is that under irradiation, instead of dissolving the precipitates coarsen. This fenomena cause the material to soften to some degree [6]. The conclusions that can be drawn from Zhang et al. [6] is that hardness increases with segregation of impurities in RPV steels, one of them is copper. Their binding to point defects in the matrix furthers the movement of these $\mathrm{Cu}$-rich precipitate clusters. These clusters migrate to the areas of low energy such as grain boundaries, and sometimes they act as 'sinks' to which point defects 
continue to migrate. This process means that these sinks can also act as recombination sites, where interstitials and vacancies can combine and 'recover' the hardness of the material by some fraction. This process can occur during irradiation and promotes improvement of the mechanical properties of the material.

Further study conducted by Shibamoto et al. also suggests that hardening is caused by copper clustering in the matrix, and that nickel additions contribute to this mechanism. Their work agrees that Frenkel pairs increase hardening; however, they also suggest that after annealing above $400{ }^{\circ} \mathrm{C}$, microvoids are no longer a cause of hardening, as they disappear above $200{ }^{\circ} \mathrm{C}$ [7].

Hardness is the capability of a substance to resist permanent shape change under applied strain. Historically, it has been defined as a material's ability to resist scratching from other materials. Many different methods for measuring hardness have been developed over the years, all of which have their own intricacies [8,9]. The Vickers hardness test has the advantage of very high versatility, for example, that it can be used on both the micro and macro scale [10,11]. The Vickers hardness test was the technique employed in this research. In this paper, TRIM is used to explore the irradiation effect on high and low copper. Moreover, this program is also useful in discovering the penetration depth of proton beams on high and low copper. By finding the penetration depth of protons, a suitable load for the Vickers hardness test can be selected.

\section{MATERIALS AND METHODS}

Microhardness for high and low copper samples was measured using a Vickers hardness machine. The indent was a diamond in the form of a square-based pyramid. The employed angle of the indent was $136^{\circ}$. As generally known, hardness is a measure of force per area $[12,13]$. Hardness can be calculated by:

$$
d=\sqrt{\frac{1.8544 * F}{H V}}
$$

Additionally, it is possible to work out the indent depth:

$$
h=\frac{d}{7.0006}
$$

where $H V$ is hardness value, $d$ is dimeter of the indentation, $F$ is load and $h$ is indenter depth.
The Stopping and Range of Ions in Matter (SRIM) and the Transport of Ions in Matter (TRIM), or SRIM-TRIM, is a Monte Carlo simulation code for the measurement of the stopping and range of ions into target matter by using a full quantum mechanical treatment of ion-atom collision (assuming a transferring atom as an ion, as well as all target atoms as an atom) [14]. Furthermore, this code can be used to gain information regarding the penetration depth of the ions into the surface of materials [15].

In this work, the type of TRIM Calculation used was the Ion Distribution and Quick Calculation of Damage, which is based on Kinchin-Pease model. The ion used was Hydrogen, which can perfectly simulate the effects of a proton. One target layer with 9 different elements was used as shown in Table 1. With the intention to simulate different compositions, the low and high copper ones, the atomic percent values of the elements were input. The simulation was performed for both compositions with ions of energies of 1,3 and $9 \mathrm{MeV}$.

\begin{tabular}{|c|c|c|c|c|c|c|c|c|c|}
\hline & \multicolumn{9}{|c|}{ Element } \\
\hline & $\mathrm{Fe}$ & C & $\mathrm{Si}$ & $\mathrm{Mn}$ & P & $\mathrm{Cr}$ & Mo & $\mathrm{Ni}$ & $\mathrm{Cu}$ \\
\hline Low copper & 93.2 & 1.15 & 0.4 & 1.5 & 0.01 & 0.1 & 0.3 & 3.3 & 0.04 \\
\hline High copper & 93.2 & 0.93 & 0.4 & 1.5 & 0.01 & 0.1 & 0.3 & 3.3 & 0.26 \\
\hline
\end{tabular}

Table 1. Compositions of low and high copper (in at\%).

The Monte Carlo code TRIM was used to gain information about the displacement per atom into the surface of materials. Displacement per atom is a strong function to measure the amount of radiation damage in irradiated materials [16]. The ion used was hydrogen with ions of energies of 1,3 and $9 \mathrm{MeV}$ primary knock-on atom (PKA) into high and low copper for 5,000 incident ions

One sample of each composition was irradiated by $3 \mathrm{MeV}$ protons. The beam current was $5 \mu \mathrm{A}$ and the samples were irradiated for approximately 2 hours. The total charge applied was $36,000 \mu \mathrm{C}$. The temperature was controlled by cooled water and kept at approximately $30{ }^{\circ} \mathrm{C}$. The beam area on each sample was $25 \mathrm{~mm}^{2}$. An additional irradiation was performed with the same parameters except the temperature. The temperature was kept higher, at approximately $380{ }^{\circ} \mathrm{C}$. Unfortunately, a contamination in the vacuum system caused a black layer on the surface, which invalidated the samples damaged at temperature. A Scanning Electron Microscope (SEM) test was done to identify the elements, but regrettably it was not possible to test these two specimens in the Vickers Hardness test. 
Hardness tests were performed on the high and low copper samples irradiated at $30{ }^{\circ} \mathrm{C}$ as described before. It was not possible to continue with the hardness tests because the black layer on the surface had a brittle profile. The indents were causing cracks in the material so the result was not valid because Vickers Hardness test is not supposed to be performed in brittle surfaces. Figure 1 shows the photos of the invalid specimens.

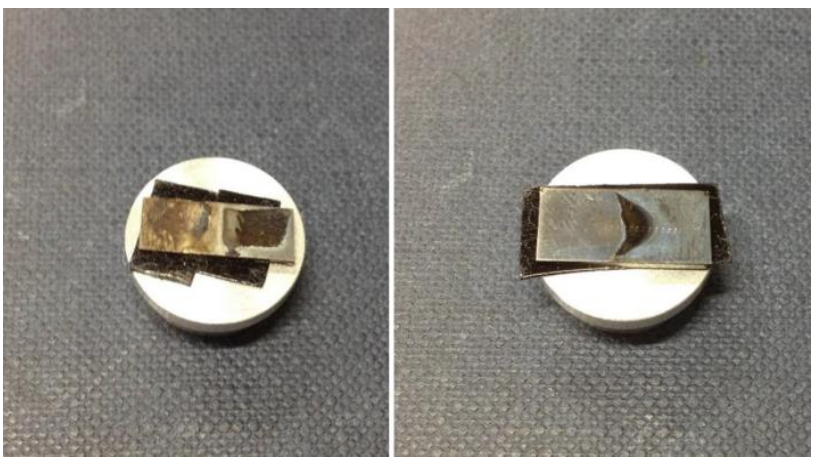

Fig. 1. A contamination in the vacuum system has invalided specimens irradiated at $380^{\circ} \mathrm{C}$.

Different loads were used in order reach different indent depths in the sample. Three $\mathrm{MeV}$ protons can reach up to approximately $40 \mu \mathrm{m}$ in the samples. Vickers Hardness test is able to measure hardness up to $25 \mu \mathrm{m}$, and a good simulation of neutron damage profile can be obtained. The indent depths for different loads were approximately $3,8,11$ and $16 \mu \mathrm{m}$. Indents were 0.5 $\mathrm{mm}$ apart from each other to avoid interference in the measurements. Moreover, measurement started and ended far away from the edges of the specimens.

\section{RESULTS AND DISCUSSION}

One of the greatest advantages of using proton beam is the easy variation of parameters. Accelerators, such as the cyclotron used in this work, offer a huge variety of irradiation environments. It is important to express the displacements in terms of ion/ $/ \mathrm{cm}^{2}$ because this value can be easily changed for different irradiation conditions. The graphs for low copper compositions are shown in Fig. 2. The results were satisfactory as the dpa (displacement per atom) behaviour is clear. For higher energies, the dose at the surface decreases because they pass through the material easily, so the scattering cross section is lower. Moreover, the results were of the same pattern for high copper.

For the TRIM calculation of proton penetration in low copper, the proton penetration depth varies strongly with its energy, and no difference was observed between low and high copper compositions. The penetration depth was approximately 7,38 and $234 \mu \mathrm{m}$ respective for 1,3 and $9 \mathrm{MeV}$ protons.

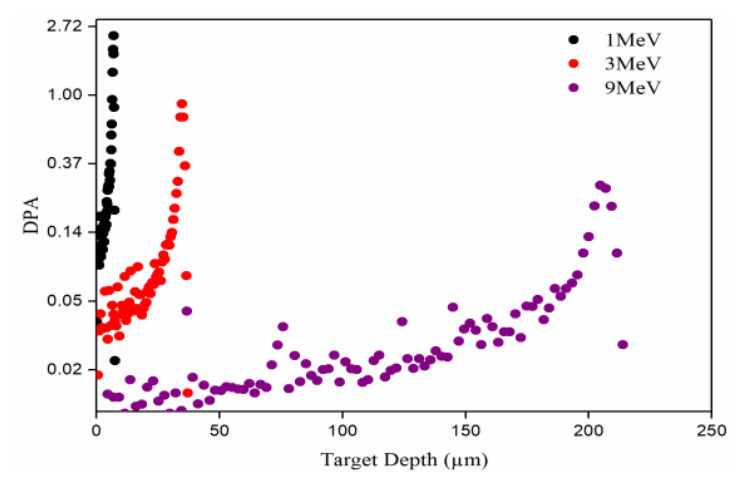

Fig. 2. Bragg peak in low copper, showing target depth change with increase displacement per atom at 1,3 and $9 \mathrm{MeV}$ proton.

Details of hardness results have made it possible to notice that it does not have the same number of points for each condition. This is because the work was held throughout the surface and the interface between non irradiated and irradiated sides was not exactly in the half of the row. Additionally, the indent depth was approximately the same for all specimens and conditions, so this value was also averaged in order to make comparisons easier. The study found that the influence of Copper in the specimen was noticable. It was slighter than expected. The surface was considerably rough while doing the polishing was complex due to safety issues related to the residual activity of the samples. As shown in Fig. 3(c), the surface of the sample in what assumed to be the iraddiated zones appears more dirty than the surface shown in Fig. 3(a) and (b).

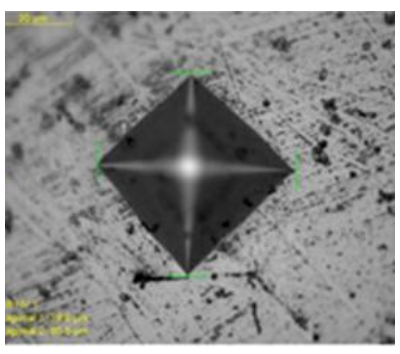

(a)

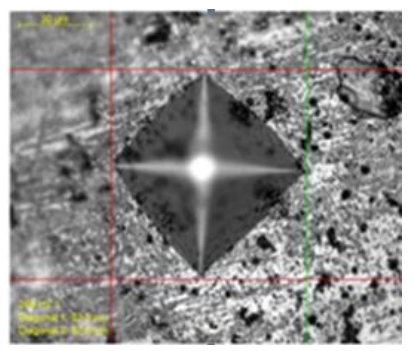

(b)

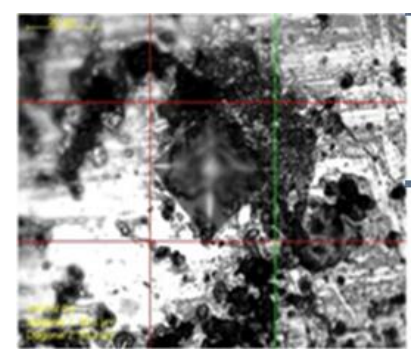

(c)

Fig. 3. Images from the high copper hardness analysis. Indent on the non-irradiated surface (a), Indent on the irradiated surface (b), Example of invalid indent (c). 
Different depths on the material receive different damage accumulation due their different volumes. The deeper penetration, the greater dose is received. The hardness profile through sample thickness can be seen in Figs. 4 and 5. The values are averages for the non-irradiated and irradiated parts for both samples and the error bars express the standard deviation. Figures 4 and 5 show that irradiated values are slightly higher than nonirradiated values.

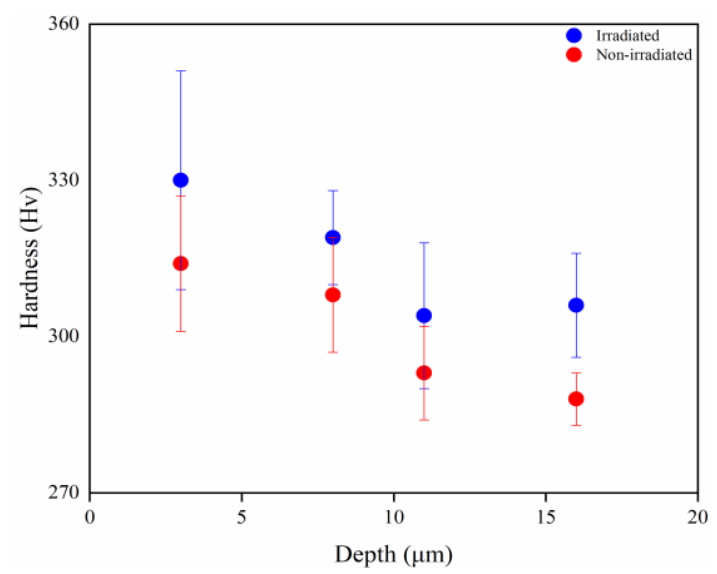

Fig. 4. Hardness versus depth for non-irradiated and irradiated low copper.

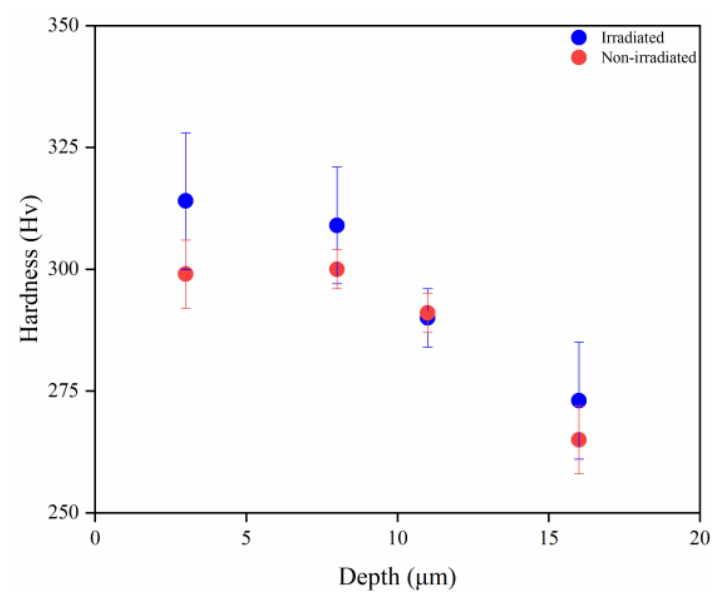

Fig. 5. Hardness versus depth for non-irradiated and irradiated high copper.

The profile was not the best expected because hardness, which is represented by depth, should increase with dose. However, it is noticable that the standard deviation is large and ranging a great variation of values for both samples. In fact, the surfaces were not ideal to do hardness tests because they were rough.

The hardening result from proton irradiation is shown in terms of yield strength in Figs. 6 and 7. It was calculated from hardness results using the following relation $[17,18]$ :

$$
\Delta \sigma_{y}=3.55 \Delta H V
$$

where $\Delta \sigma_{y}$ is the increment in yield strength and $\triangle H V$ is the increment in hardness.

Was et al. [19] have conducted a calculation of the yield strength variation for protons and neutrons irradiations for two different types of austenitic steels and higher doses of 1-6 dpa. Their results are in excellent agreement, and the increase of yield strength with dose is clear for each experiment. The increase of yield strength with dose is anticipated. The errors bars in Figs. 6 and 7 represent the standard deviation for yield strength average values, and they cover a large variation of values.

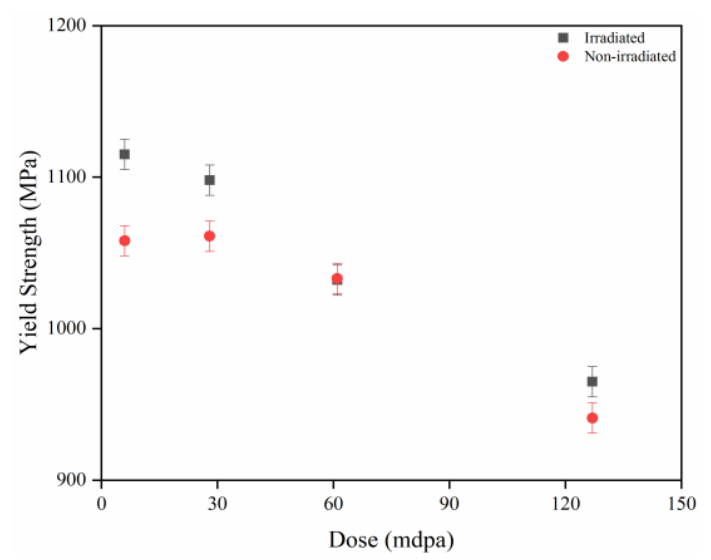

Fig. 6. Yield Strength versus Dose for non-irradiated and irradiated high copper.

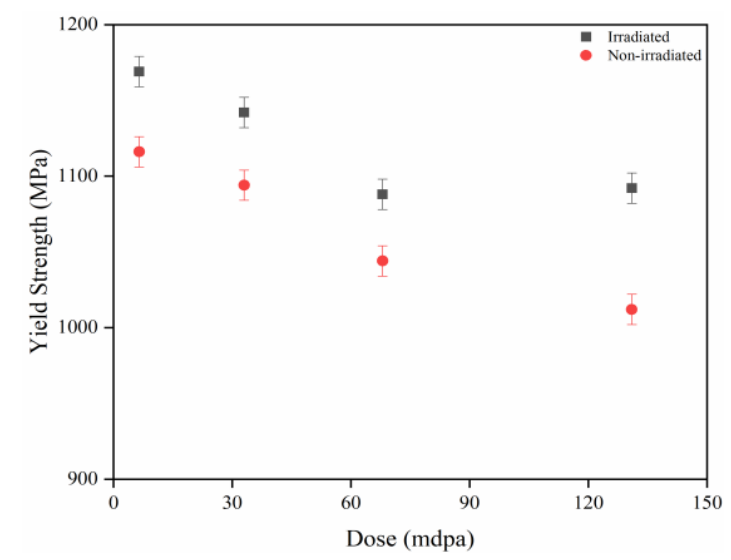

Fig. 7. Yield Strength versus Dose for non-irradiated and irradiated low copper

The yield strength variation through depth is not in great agreement with other experiments. However, the dose applied in this experiment was much lower $\sim 150 \mathrm{mdpa}$. It is expected to have better results and cleaner profiles with higher doses. Additionally, the surfaces of the samples were not very smooth and shiny as expected for a hardness test. 


\section{CONCLUSION}

Nano indentation experiments were performed to invistigate the effect of ion irradiation on the hardness properties of high and low copper. Irradiated values for hardness were higher than nonirradiated ones as expected from radiation hardening. Furthermore, it was expected that high copper samples had a higher increase in hardness. This copper influence was not very perceptive in the results.

The hardness results in this work did not satisfy the predictions, where the values had great variation resulting in high standard deviations for the averages. The study found that the total dose in the first 25 microns of the flat damage profile were $0.367 \mathrm{dpa}$ for low copper and $0.373 \mathrm{dpa}$ for high copper samples. The hardness results appear to be clearer for higher doses, such as 1-6 dpa. Also, the surfaces were rough and not ideal for hardness tests.

\section{ACKNOWLEGMENT}

The author would like to thank the Ministry of Higher Education and Scientific Research / Kurdistan of Iraq for the financial support and providing the facilities for this study.

\section{REFERENCE}

1. C.D. Hardie, C.A. Williams, S. Xu et al., J. Nucl. Mater.439 (2013) 33.

2. H.M. Qadr and A.M. Hamad, Scientific Bulletin of Valahia University-Materials and Mechanics 17 (2019) 18.

3. H.M. Qadr, International Journal of Nuclear Energy Science and Technology 13 (2019) 61.

4. G.S. Was, Fundamentals of Radiation Materials Science: Metals and Alloys, $2^{\text {nd }}$ ed., Springer, New York (2017).

5. J. Zelenty, Mater. Sci. Technol. 31 (2015) 981.

6. Z. Zhang, C.T. Liu, M. Miller et al., Acta Mater. 60 (2012) 3034.

7. H. Shibamoto, A. Kimura, M. Hasegawa et al., Journal of ASTM International 2 (2005) 1.

8. M. Abdel-Rahman, A. Aldeen Ahmed and E.A. Badawi, Testing Natural Aging Effect on Properties of 6066 \& 6063 Alloys Using Vickers Hardness and Positron Annihilation Lifetime Techniques, Defect and Diffusion Forum, Trans. Tech. Publ. (2010) 107.

9. D. Tabor, Philosophical Magazine A $\mathbf{7 4}$ (1996) 1207.

10. E. Broitman, Tribology Letters 65 (2017) 23.

11. A. Jahja, N. Effendi and M. Dani, Atom Indonesia 33 (2007) 79.

12. H.M. Qadr and A.M. Hamad, Problems of Atomic Science and Technology, Series Thermonuclear Fusion 42 (2019) 81.

13. H.M. Qadr, Annals of the University of Craiova Physics AUC 29 (2019) 68.

14. R.E. Stoller, M.B. Toloczko, G.S. Was et al., Nuclear Instruments and Methods in Physics Research Section B: Beam Interactions with Materials and Atoms 310 (2013) 75.

15. J.F. Ziegler, M.D. Ziegler and J.P. Biersack, Nuclear Instruments and Methods in Physics Research Section B: Beam Interactions with Materials and Atoms 268 (2010) 1818.

16. E.A. Read, H.R. Trellue and C.R.E. de Oliveir, Assessment of Three-Dimensional Monte Carlo Burnup for Gas Cooled Reactors Using the Reactivity Equivalent Physical Transformation Method, International Conference on Mathematics and Computational Methods Applied to Nuclear Science and Engineering (M\&C 2011) 1.

17. Q. Dong, H. Qin, Z. Yao and M.R. Daymond, Materials \& Design 161 (2019) 147.

18. J.T. Busby, M.C. Hash and G.S. Was, J. Nucl. Mater. 336 (2005) 267.

19. G. Was, J. Busby, T. Allen et al., J. Nucl. Mater. 300 (2002) 198. 